\title{
ON THE STABLE RANK OF ALGEBRAS OF OPERATOR FIELDS OVER AN N-CUBE
}

\author{
PING WONG NG AND TAKAHIRO SUDO
}

\begin{abstract}
Let $\mathcal{A}$ be a unital maximal full algebra of operator fields with base space $[0,1]^{k}$ and fibre algebras $\left\{\mathcal{A}_{t}\right\}_{t \in[0,1]^{k}}$. We show that the stable rank of $\mathcal{A}$ is bounded above by the quantity $\sup _{t \in[0,1]^{k}} \operatorname{sr}\left(C\left([0,1]^{k}\right) \otimes\right.$ $\mathcal{A}_{t}$ ). Here the symbol "sr" means stable rank. Using the above estimate, we compute the stable ranks of the $C^{*}$-algebras of the (possibly higher rank) discrete Heisenberg groups.
\end{abstract}

\section{INTRODUCTION}

Rieffel [12] introduced the notion of stable rank for $C^{*}$-algebras as the noncommutative version of complex dimension of ordinary topological spaces. It turns out that the stable rank of a unital $C^{*}$-algebra is the same as its Bass stable rank (see [7]). The purpose of this paper is to study stable rank for continuous field $C^{*}$-algebras. Our main result is

Theorem 1.1. Let $\mathcal{A}$ be a unital maximal full algebra of operator fields with base space $[0,1]^{k}$ and fibre algebras $\left\{\mathcal{A}_{t}\right\}_{t \in[0,1]^{k}}$. Then the stable rank of $\mathcal{A}$ satisfies the following inequality:

$$
\operatorname{sr}(\mathcal{A}) \leq \sup _{t \in[0,1]^{k}} \operatorname{sr}\left(C\left([0,1]^{k}\right) \otimes \mathcal{A}_{t}\right) .
$$

One of the key ingredients of the proof of the above result is Nistor's notion of absolute connected stable rank (see [10]). Recall that for a unital $C^{*}$-algebra $\mathcal{A}$, the absoluted connected stable rank of $\mathcal{A}$ is numerically the same as the stable rank of the tensor product $C[0,1] \otimes \mathcal{A}$.

As an application of our theorem, we will compute the stable ranks of the universal $C^{*}$-algebras of the (possibly higher rank) discrete Heisenberg groups. Recall that for a positive integer $n$, the discrete Heisenberg group of rank $2 n+1$ is the group of all $2 n+1$ by $2 n+1$ upper triangular matrices with integer entries, with ones on the diagonal, and zero entries on all but the first row, last column and the diagonal. The rank $2 n+1$ discrete Heisenberg group is naturally a lattice subgroup of the $2 n+1$-dimensional Heisenberg Lie group.

The stable ranks of the universal $C^{*}$-algebras of various type I Lie groups have been extensively studied (see [13], [15], [16]). In particular, we point out that for a simply connected nilpotent Lie group $G$, the stable rank of the universal $C^{*}$-algebra $C^{*}(G)$ of $G$ has been computed by Sudo and Takai (see [15]). Roughly speaking, they showed that the stable rank of $C^{*}(G)$ is controlled by the ordinary topological dimension of the space of one-dimensional representations of $G$.

Recently, the stable ranks of a class of non-type I solvable Lie groups (which include the Mautner group) have also been computed (see [14]).

Our computations for the discrete Heisenberg groups constitute a class of interesting nontrivial examples of the stable rank of the universal $C^{*}$-algebra of a non-type I discrete group.

In a later paper, we will apply the techniques developped in this paper to compute the stable ranks of arbitrary finitely generated, torsion-free two-step nilpotent groups.

A general reference for stable rank of $C^{*}$-algebras is [12]. General references for algebras of operator fields are $[\mathbf{5}],[\mathbf{8}]$ and $[\mathbf{1 7}]$.

In what follows, for a $C^{*}$-algebra $\mathcal{A}$, the notation " $\operatorname{sr}(\mathcal{A})$ " will always mean the stable rank of $\mathcal{A}$. If, in addition, $\mathcal{A}$ is unital and $N$ is a positive integer, then $L g_{N}(\mathcal{A})$ is the set of $N$-tuples $\left(a_{1}, a_{2}, \ldots, a_{N}\right)$ in $\mathcal{A}^{N}$ such that $\sum_{i=1}^{N}\left(a_{i}\right)^{*} a_{i}$ is an invertible element in $\mathcal{A}$.

Date: November 4, 2018.

Mathematics subject classification: 47L99. 


\section{MAIn RESULTS}

Lemma 2.1. Let $\mathcal{A}$ be a unital maximal full algebra of operator fields with base space [0,1] and fibre algebras $\left\{\mathcal{A}_{t}\right\}_{t \in \mathcal{F}}$. Then the stable rank of $\mathcal{A}$ satisfies the inequality

$$
\operatorname{sr}(\mathcal{A}) \leq \sup _{t \in[0,1]} \operatorname{sr}\left(C[0,1] \otimes \mathcal{A}_{t}\right) .
$$

Proof. In this proof, we will always let " $\mathcal{F}$ " denote the continuity structure for the continuous field decomposition of $\mathcal{A}$ in the hypothesis.

Suppose that $M=\sup _{t \in[0,1]} \operatorname{sr}\left(C[0,1] \otimes \mathcal{A}_{t}\right)$ is a finite number. Let an $M$-tuple $\left(a_{1}, a_{2}, \ldots, a_{M}\right) \in \mathcal{A}^{M}$ and a positive real number $\epsilon>0$ be given. Since $[0,1]$ is compact, let $I_{1}, I_{2}, \ldots, I_{N}$ be a finite set of open intervals which cover $[0,1]$ and for each $i=1,2, \ldots, N$, let $\left(f_{i, 1}, f_{i, 2}, \ldots, f_{i, M}\right)$ be an $M$-tuple in $\mathcal{A}^{M}$ such that

1. $\sum_{j=1}^{M} f_{i, j}(t)^{*} f_{i, j}(t)$ is an invertible element of $\mathcal{A}_{t}$ for $t \in I_{i}$. Here the operator field $t \mapsto f_{i, j}(t)$ for $t \in[0,1]$, is the representation of $f_{i, j}$ as a continuous operator field in $\mathcal{A}$. And

2. $\left\|f_{i, j}-a_{j}\right\|<\epsilon$, for all $i, j$.

We can choose such intervals $I_{i}$ and such elements $f_{i, j}$, since our hypothesis implies that the stable rank of each fibre $\mathcal{A}_{t}$ is less than or equal to $M$, and by the existence and continuity of operator fields in a full algebra of operator fields (see the definition of full algebra of operator fields in [5], [8] or [17]). Also, one needs to use the fact that any element of a unital $C^{*}$-algebra that is sufficiently close to the unit is invertible. Finally, to make $f_{i, j}$ uniformly within $\epsilon$ of $a_{j}$ (for all $i, j$ ), one needs the maximality of the algebra of operator fields $\mathcal{A}$ (see [8] Proposition 1 and [17] Theorem 1.1).

For simplicity, we will assume that there are only two intervals $\left(I_{1}\right.$ and $\left.I_{2}\right)$ and only two $M$-tuples ( $\left(f_{1,1}, f_{1,2}, \ldots, f_{1, M}\right)$ and $\left.\left(f_{2,1}, f_{2,2}, \ldots, f_{2, M}\right)\right)$. We may additionally assume that neither interval is contained in the closure of the other, and that their intersection is a continuum. Our goal is to "connect" the two $M$ tuples over the intersection $\overline{I_{1} \bigcap I_{2}}$ to get an element of $\operatorname{Lg}_{M}(\mathcal{A})$ which approximates $\left(a_{1}, a_{2}, \ldots, a_{M}\right)$ within $\epsilon$. The proof for more than two intervals is an iteration of this procedure (after appropriate contraction, removal or addition of intervals...).

Our procedure for "connecting" the two $M$-tuples over $\overline{I_{1} \bigcap I_{2}}$ will involve constructing a sequence of strictly increasing points $\left\{t_{n}\right\}_{n=1}^{\infty}$ in $I_{1} \cap I_{2}$, and constructing sequences of operator fields $\left\{\alpha_{j}^{n}\right\}_{n=1}^{\infty}(j=$ $1,2, \ldots, M)$ satisfying:

1. $\alpha_{j}^{n}$ is a continuous operator field in $\mathcal{A}(n)$, for all $j, n$. Here $\mathcal{A}(n)$ is the unital maximal full algebra of operator fields gotten by restricting $\mathcal{A}$ to the interval $\left[t_{n}, t_{n+1}\right]$. (In particular, the continuity structure for $\mathcal{A}(n)$ is gotten by taking the restriction to $\left[t_{n}, t_{n+1}\right]$ of all the fields in $\mathcal{F}$ ).

2. $\alpha_{j}^{n}$ is within $\epsilon$ of the restricion of $a_{j}$ to $\left[t_{n}, t_{n+1}\right]$, for all $j, n$.

3. $\alpha_{j}^{1}\left(t_{1}\right)=f_{1, j}\left(t_{1}\right)$ and $\alpha_{j}^{n+1}\left(t_{n+1}\right)=\alpha_{j}^{n}\left(t_{n+1}\right)$, for all $j, n$.

4. $\alpha_{j}^{n+1}$ is within $\epsilon / 2^{n}$ of the restriction of $f_{2, j}$ to $\left[t_{n+1}, t_{n+2}\right]$, for all $j, n$. And

5. $\left(\alpha_{1}^{n}, \alpha_{2}^{n}, \ldots, \alpha_{M}^{n}\right)$ is in $L g_{M}(\mathcal{A}(n))$; or equivalently, $\sum_{j=1}^{M} \alpha_{j}^{n}(t)^{*} \alpha_{j}^{n}(t)$ is an invertible element of $\mathcal{A}_{t}$ for all $t \in\left[t_{n}, t_{n+1}\right]$, for all $n$ (the proof of equivalence is a small spectral theory argument which uses the fact that $\left[t_{n}, t_{n+1}\right]$ is compact).

Henceforth, we will let "(*)" denote conditions (1) - (5).

By [10] Lemma 2.4 and our hypothesis for $M$, we have that $L g_{M}\left(\mathcal{A}_{t}\right) \bigcap\left\{\left(b_{1}, b_{2}, \ldots, b_{M}\right) \in \mathcal{A}_{t}^{M}: \| a_{i}(t)-\right.$ $\left.b_{i} \|<\epsilon \forall i\right\}$ is a nonempty connected open set for all $t \in[0,1]$. Hence, fixing $t_{1} \in I_{1} \bigcap I_{2}$, there are continuous paths $\gamma_{j}:[0,1] \rightarrow \operatorname{Lg}_{M}\left(\mathcal{A}_{t_{1}}\right), j=1,2, \ldots, M$, such that a) $\gamma_{j}(0)=f_{1, j}\left(t_{1}\right)$ and $\gamma_{j}(1)=f_{2, j}\left(t_{1}\right)$ for all $j$, and b) $\gamma_{j}(s)$ is within $\epsilon$ of $a_{j}\left(t_{1}\right)$ for all $s \in[0,1]$ and for all $j$.

Note that since $[0,1]$ is compact, $\left(\gamma_{1}, \gamma_{2}, \ldots, \gamma_{M}\right)$ is in $\operatorname{Lg}_{M}\left(C[0,1] \otimes \mathcal{A}_{t_{1}}\right)$. Now consider the unital maximal full algebra of operator fields $\widetilde{\mathcal{A}}$, which has base space $[0,1]$ and fibre algebras $\left\{C[0,1] \otimes \mathcal{A}_{t}\right\}_{t \in[0,1]}$. The continuity structure $\widetilde{\mathcal{F}}$ for $\widetilde{\mathcal{A}}$ consists of all operator fields of the form $t \mapsto \sum_{i=1}^{N} f_{i} \otimes c_{i}(t), t \in[0,1]$. Here the $f_{i}$ s are in $C[0,1]$, the $c_{i}$ s are continuous operator fields in $\mathcal{A}$ (with respect to the continuity structure $\mathcal{F}$ ), and $N$ is a nonnegative integer. Now since $\widetilde{\mathcal{A}}$ is a full algebra of operator fields, there are continuous operator fields $t \mapsto \gamma_{j}(., t)$ in $\widetilde{\mathcal{A}}$ such that $\gamma_{j}\left(., t_{1}\right)=\gamma_{j}(),. j=1,2, \ldots, M$. (Here, the second variable of $\gamma_{j}(.,$.$) ranges$ over the base space of $\widetilde{\mathcal{A}}$, and for $t \in[0,1], \gamma_{j}(., t)$ is an element of the fibre algebra $C[0,1] \otimes \mathcal{A} t$ of $\left.\widetilde{\mathcal{A}}\right)$.

One can show that the operator field $t \mapsto \gamma_{j}(1, t)$ is a continuous operator field in $\mathcal{A}$ (with continuity structure $\mathcal{F}$ ) for $j=1, \ldots, M$. Also, one may view the field $t \mapsto a_{j}(t)$ as an operator field in $\widetilde{\mathcal{A}}$ in the natural 
way for $j=1, . ., M$. From these and the fact that (as elements of $\left.C[0,1] \otimes \mathcal{A}_{t_{1}}\right) \gamma_{j}\left(., t_{1}\right)$ is within $\epsilon$ of $a_{j}\left(t_{1}\right)$, we can find an open neighbourhood $V$ of $t_{1}$ with $V \subset I_{1} \cap I_{2}$ such that for all $t \in V$, a) as elements of $C[0,1] \otimes \mathcal{A}_{t}, \gamma_{j}(., t)$ is within $\epsilon$ of $a_{j}(t), j=1,2, \ldots, M$, and b) $\gamma_{j}(1, t)$ is within $\epsilon / 2$ of $f_{2, j}(t)$. Furthermore, by continuity and since elements in a unital $C^{*}$-algebra which are sufficiently close to the unit are invertible, we may assume that $V$ is sufficiently small so that for all $t \in V, \sum_{j=1}^{M} \gamma_{j}(., t)^{*} \gamma_{j}(., t)$ is an invertible element of $C[0,1] \otimes \mathcal{A}_{t}$.

One can show that for any continuous function $g:[0,1] \rightarrow[0,1]$, the operator field $t \mapsto \gamma_{j}(g(t), t)$ is a continuous field in $\mathcal{A}$, for $j=1,2, \ldots, M$. Hence, we can pick $t_{2} \in V$ such that $t_{1}<t_{2}$; and we can let $\alpha_{j}^{1}(t)=_{d f} \gamma_{j}\left(\left(t-t_{1}\right) /\left(t_{2}-t_{1}\right)\right)$ for all $t \in\left[t_{1}, t_{2}\right], j=1,2, . ., M$. This will give us the first members of the sequences in $(*)$.

Now we can repeat almost exactly the same argument as before, replacing $t_{1}$ by the point $t_{2}$ and replacing $f_{1, j}\left(t_{1}\right)$ by $\alpha_{j}^{1}\left(t_{2}\right), j=1,2, \ldots, M$. In this manner, we get a point $t_{3}$ and operator fields $\alpha_{j}^{2}, j=1,2, \ldots, M$, which will be the next members of the sequences in $(*)$. We need only note two minor modifications that are needed in the argument: a) firstly, one has to use the fact that for all $t$, the set

$$
L g_{M}\left(\mathcal{A}_{t}\right) \bigcap\left\{\left(b_{1}, b_{2}, \ldots, b_{M}\right) \in \mathcal{A}_{t}^{M}:\left\|a_{j}(t)-b_{j}\right\|<\epsilon \text { and }\left\|f_{2, j}(t)-b_{j}\right\|<\epsilon / 2, \text { for all } j\right\}
$$

is a connected open set (see [10] Lemma 2.4), and b) when choosing the corresponding neighbourhood about $t_{2}$, one must make it sufficiently small so that the corresponding quantities which result will also be sufficiently small in order to fulfill condition (4) in $\left(^{*}\right)$.

Repeating this process ad infinitum (making the appropriate modifications at each step), we get a sequence of points $\left\{t_{n}\right\}_{n=1}^{\infty}$ and sequences of continuous operator fields $\left\{\alpha_{j}^{n}\right\}_{n=1}^{\infty}, j=1,2, \ldots, M$, which fulfill the conditions in $(*)$. Now let $\tilde{t}=\lim _{n \rightarrow \infty} t_{n}$. For $j=1,2, \ldots, M$, let $\alpha_{j}$ be the continuous operator field in $\mathcal{A}$ defined by

1. $\alpha_{j}(t)=f_{1, j}(t)$ for $t \in\left[0, t_{1}\right]$,

2. $\alpha_{j}(t)=\alpha_{j}^{n}(t)$ for $t \in\left[t_{n}, t_{n+1}\right]$, and

3. $\alpha_{j}(t)=f_{2, j}(t)$ for all $t \in[\tilde{t}, 1]$.

Then $\left(\alpha_{1}, \alpha_{2}, \ldots, \alpha_{M}\right) \in L g_{M}(\mathcal{A})$, and for all $j=1,2, \ldots, M, \alpha_{j}$ approximates $a_{j}$ within $\epsilon$.

Proof of Theorem 1.1. We proceed by induction. The base case $k=1$ has already been dealt with in Lemma 2.1. We now do the induction step, supposing that $k \geq 2$. By [8] Theorem 4 , let $\pi: \operatorname{Prim}(\mathcal{A}) \rightarrow[0,1]^{k}$ be the continuous open surjection corresponding to the continuous field decomposition of $\mathcal{A}$ in the hypothesis $($ Here $\operatorname{Prim}(\mathcal{A})$ is the primitive ideal space of $\mathcal{A})$.

Since $k \geq 2$, the map $p:[0,1]^{k} \rightarrow[0,1]^{k-1}$, given by projecting onto the first $k-1$ coordinates, is a continuous open surjection. Hence, the composition $p \circ \pi: \operatorname{Prim}(\mathcal{A}) \rightarrow[0,1]^{k-1}$ is a continuous open surjection. Hence by $[\mathbf{8}]$ Theorem 4 , we can realize $\mathcal{A}$ as a unital maximal full algebra of operator fields with base space $[0,1]^{k-1}$ and fibre algebras, say, $\left\{\mathcal{B}_{s}\right\}_{s \in[0,1]^{k-1}}$. Hence by the induction hypothesis, $\operatorname{sr}(\mathcal{A}) \leq$ $\sup _{s \in[0,1]} \operatorname{sr}\left(C\left([0,1]^{k-1}\right) \otimes \mathcal{B}_{s}\right)$.

But for each $s$, the fibre algebra $\mathcal{B}_{s}$ can be realized as a maximal full algebra of operator fields with base space $p^{-1}(s)=\{s\} \times[0,1]$ and fibre algebras $\left\{\mathcal{A}_{r}\right\}_{r \in\{s\} \times[0,1]}$ (By [8] Theorem 4, $\mathcal{B}_{s}$ is isomorphic to $\mathcal{A} / I$ where $I=\bigcap(p \circ \pi)^{-1}(s)$. Using this fact, one can construct the natural continuous open surjection of $\operatorname{Prim}\left(\mathcal{B}_{s}\right)$ onto $\left.\{s\} \times[0,1]\right)$. Let us suppose that this continuous field decomposition of $\mathcal{B}_{s}$ is given by a continuity structure $\mathcal{G}$. Then $C\left([0,1]^{k-1}\right) \otimes \mathcal{B}_{s}$ can be realized as a unital maximal full algebra of operator fields with base space $[0,1]$ and fibre algebras $\left\{C\left([0,1]^{k-1}\right) \otimes \mathcal{A}_{r}\right\}_{r \in\{s\} \times[0,1]}$. Here the continuity structure consists of operator fields of the form $r \mapsto \sum_{i=1}^{N} f_{i} \otimes b_{i}(r)$, for $r \in\{s\} \times[0,1]$. Here the $f_{i}$ s are in $C\left([0,1]^{k-1}\right)$, the $b_{i}$ s are continuous fields in $\mathcal{B}_{s}$ (with respect to the continuity structure $\mathcal{G}$ ) and $N$ is a nonnegative integer.

Hence by the induction hypothesis, for each $s$, we have that $\operatorname{sr}\left(C\left([0,1]^{k-1}\right) \otimes \mathcal{B}_{s}\right) \leq \sup _{r \in\{s\} \times[0,1]} \operatorname{sr}\left(C\left([0,1]^{k}\right) \otimes\right.$ $\left.\mathcal{A}_{r}\right)$. It follows, then, that $\operatorname{sr}(\mathcal{A}) \leq \sup _{t \in[0,1]^{k}} \operatorname{sr}\left(C\left([0,1]^{k}\right) \otimes \mathcal{A}_{t}\right)$.

We note that the statements of Lemma 2.1 and Theorem 1.1 would still be true if the unit interval $[0,1]$ was replaced by the circle $S^{1}$ (and if the $k$-cube $[0,1]^{k}$ was replaced by the $k$-torus $\mathbb{T}^{k}$ ). The proofs would be exactly the same. In other words, we have that 
PING WONG NG AND TAKAHIRO SUDO

Corollary 2.2. Let $\mathcal{A}$ be a unital maximal full algebra of operator fields with base space the $k$-torus $\mathbb{T}^{k}$ and fibre algebras $\left\{\mathcal{A}_{t}\right\}_{t \in \mathbb{T}^{k}}$. Then the stable rank of $\mathcal{A}$ satisfies

$$
\operatorname{sr}(\mathcal{A}) \leq \sup _{t \in \mathbb{T}^{k}} \operatorname{sr}\left(C\left([0,1]^{k}\right) \otimes \mathcal{A}_{t}\right)
$$

Theorem 2.3. Let $H_{2 n+1}^{\mathbb{Z}}$ be the discrete Heisenberg group of rank $2 n+1$. Let $C^{*}\left(H_{2 n+1}^{\mathbb{Z}}\right)$ be the univeral $C^{*}$-algebra of $H_{2 n+1}^{\mathbb{Z}}$. Then $\operatorname{sr}\left(C^{*}\left(H_{2 n+1}^{\mathbb{Z}}\right)\right)=n+1$.

Proof. By [12] Proposition 1.7 and Theorem 4.3, and the fact that $C\left(\mathbb{T}^{2 n}\right)$ is a quotient of $C^{*}\left(H_{2 n+1}^{\mathbb{Z}}\right)$, the stable rank of $C^{*}\left(H_{2 n+1}^{\mathbb{Z}}\right)$ is greater than or equal to $n+1$. By [1] and [9] Theorem 3.4, $C^{*}\left(H_{2 n+1}^{\mathbb{Z}}\right)$ can be realized as a unital maximal full algebra of operator fields with base space the 1-torus $\mathbb{T}$ and fibre algebras, say, $\left\{\mathcal{A}_{t}\right\}_{t \in \mathbb{T}}$. Hence, by the Corollary 2.2, the stable rank of $C^{*}\left(H_{2 n+1}^{\mathbb{Z}}\right)$ satisfies $\operatorname{sr}\left(C^{*}\left(H_{2 n+1}^{\mathbb{Z}}\right)\right) \leq$ $\sup _{t \in \mathbb{T}} \operatorname{sr}\left(C[0,1] \otimes \mathcal{A}_{t}\right)$.

Now by [1] and [9] Theorem 3.4, each fibre algebra $\mathcal{A}_{t}$ can be realized as a unital maximal full algebra of operator fields with base space a torus with dimension less than or equal to $2 n$ (the zero-dimensional torus being a point) and fibre algebras, say, $\left\{B_{s}^{t}\right\}_{s \in \mathbb{T}^{l_{t}}}$ where $\mathbb{T}^{l_{t}}$ is the base in the continuous field decomposition of $\mathcal{A}_{t}\left(l_{t} \leq 2 n\right)$. Moreover, for each $t \in \mathbb{T}$ and for each $s \in \mathbb{T}^{l_{t}}, \mathcal{B}_{s}^{t}$ is isomorphic to either a full matrix algebra $\mathbb{M}_{n}(\mathbb{C})$ or $\mathbb{M}_{n}(\mathbb{C}) \otimes\left(\otimes^{n} \mathbb{A}_{\theta}\right)$, where $\bigotimes^{n} \mathbb{A}_{\theta}$ is the $n$ times tensor product of a fixed irrational rotation algebra $\mathbb{A}_{\theta}$ with irrational rotation $\theta$. Now by [4] each irrational rotation algebra can be decomposed as an inductive limit of building blocks of the form $M_{m}(C(\mathbb{T})) \oplus \mathbb{M}_{n}(C(\mathbb{T})$ ) (the integers $m$ and $n$ get arbitrarily large as we move along building blocks in the inductive limit). Hence, since $C[0,1] \otimes \mathcal{A}_{t}$ can be realized as a maximal full algebra of operator fields with base space $\mathbb{T}^{l_{t}}$ and fibre algebras $\left\{C[0,1] \otimes \mathcal{B}_{s}^{t}\right\}_{s \in \mathbb{T}}$, it follows, by Corollary 2.2, [12] Proposition 1.7 and Theorems 5.1 and 6.1 , that $\operatorname{sr}\left(C[0,1] \otimes \mathcal{A}_{t}\right) \leq n+1$, for all $t$. Hence, $\operatorname{sr}\left(C^{*}\left(H_{2 n+1}^{\mathbb{Z}}\right)=n+1\right.$.

\section{REFERENCES}

1 J. Anderson And W. PAschke, 'The rotation algebra', Houston J. Math., 15, (1989), 1 - 26.

2 L. BAGgett AND J. PACKer, 'The primitive ideal space of two-step nilpotent group $C^{*}$-algebras', $J$. Funct. Anal., 124, (1994), 389-426.

3 K. R. Davidison, $C^{*}$-algebras by example, Fields Institute Monographs, 6.

4 G. A. Elliott And D. E. Evans, 'The structure of the irrational rotation $C^{*}$-algebra', Ann. of Math. (2), 138, no. 3, (1993), 477-501.

5 J. M. G. Fell, 'The structure of algebras of operator fields', Acta Math., 106, (1961), 233-280.

6 N. E. Hassan, 'Rangs Stables de certaines extensions', J. London Math. Soc., (2), 52, (1995), 605-624.

7 R. Herman, L. N. Vaserstein, 'The stable rank of $C^{*}$-algebras', Invent. Math., 77, (1984), 553-555.

8 R-Y LEE, 'On $C^{*}$-algebras of operator fields', Indiana U. Math. Journal, 25, no. 4, (1976), 303-314.

9 S. T. LEE AND J. PACKER, 'Twisted group $C^{*}$-algebras for two-step nilpotent and generalized discrete Heisenberg group', J. Operator Th., 34, no. 1, (1995), 91-124.

$10 \mathrm{~V}$. NisTor, 'Stable range for the tensor products of extensions of $K$ by $C(X)$ ', J. Operator Th., 16, (1986), 387-396.

11 J. Packer and I. Raeburn, 'On the structure of twisted group $C^{*}$-algebras', Trans. AMS, 334, no. $2,(1992), 685-718$

12 M. A. Rieffel, 'Dimension and stable rank in the $K$-theory of $C^{*}$-algebras', Proc. London Math. Soc., (3), 46, (1983), 301-333.

13 T. Sudo, 'Dimension theory of group $C^{*}$-algebras of connected Lie groups of type I', J. Math. Soc. Japan, 52, no. 3, (2000), 583-590.

14 T. Sudo, 'Structure of group $C^{*}$-algebras of Lie semi-direct products $\mathbb{C} \times \mid \mathbb{R}$ ', J. Operator Theory, 46, (2001), 25-38.

15 T. SUdo AND H. TAKAI, 'Stable rank of the $C^{*}$-algebras of nilpotent Lie groups', Internat. J. Math., 6 , no. 3, (1995), 439-446.

16 T. Sudo AND H. TAKAI, 'Stable rank of the $C^{*}$-algebras of solvable Lie groups of type I', J. Operator Theory, 38, no. 1, (1997), 67-86.

17 J. Tomiyama, 'Topological representation of $C^{*}$-algebras', Tohoku Math. J., (2), 14, (1962), 187-204. 
Department of Mathematics, University of Toronto, 100 St. George St., Room 4072, Toronto, Ontario, M5S 3G3, CANADA

E-mail address: png@math.toronto.edu

Department of Mathematical Sciences, Faculty of Science, University of the Ryukyus, Nishihara-cho, Okinawa, 903-0213, JAPAN

E-mail address: sudo@math.u-ryukyu.ac.jp 